\title{
Effects of procedural variables upon reversal and interdimensional shift performance: I'
}

PEDER J. JOHNSON, AHNE FISHKIn AND LYLE E. BOURNE, JR. UNIVERSITY OF COLORADO

College Ss accomplished a reversal (R) shift faster than an interdimensional (IRD) shift over three different methods of problem presentation, when no cue cards were present. With cue cards present there was no difference between $\mathrm{R}$ and IRD shifts. The latter finding reflects an increase in the difficulty of the $R$ shift resulting from the necessity for $S$ to sort contrary to cue cards whenever a $\mathrm{R}$ shift is made.

In a previous study (Johnson, 1965) no difference in difficulty was found between $R$ and IRD shifts in solution of concept identification (CI) problems. Moreover, performance on both types of shift was affected adversely and in equal amounts by increasing the number of irrelevant stimulus dimensions. These findings are contrary to earlier research (Kendler \& Kendler, 1962) which has consistently reported better performance by adult human $S s$ on $R$ as contrasted to IRD problems. An examination of experimental procedures revealed a number of variables that might have contributed to these discrepant findings and more generally to the magnitude of R-IRD differences. It was the purpose of this experiment to investigate systematically the influence of two of these variables, viz., the problem presentation mode and the availability of cue cards.

Method

The 144 male and female undergraduates were randomly assigned to the following conditions of a factorial design: Method of Stimulus (S), Response (R) Presentation-1S-1R, 1S-2R, or 2S-1R; Cue Card-Present or Absent; Dimensions-2 or 4 irrelevant; Type of shift-R or IRD; and Problem-Color or Size dimension relevant during the preshift phase. In Condition 1S-1R and 1S2R the stimuli were presented individually; under Condition 1S-1R S was instructed to respond only to positive instances of the concept, while under Condition 1S-2R $S$ responded by placing each pattern in one of two response categories. In Condition 2S-1R two stimuli were presented on each trial and $S$ was required to indicate which of the two was correct (positive instance). With cue card present the response categories (either one or two) were identified by a sample stimulus card that contained the attribute corresponding to correct assignment of stimuli on the preshift phase of the experiment. Following the shift the cue cards were appropriate for IRD Ss, but were necessarily inappropriate for $\mathrm{R}$ Ss since the cue cards could not be changed with the shift. Either size or color was the relevant dimension for half the Ss in all groups. The change in solution for an IRD shift involved a change in the relevant dimension from color to size or from size to color. The $\mathrm{R}$ shift simply involved a reversal of the assignments of the levels on the same relevant dimension.

Each $\mathrm{S}$ was told that a series of patterns would be presented and that his task was to discover the proper response to each. Following each response $S$ was told whether or not he had responded correctly. When $S$ made 15 consecutively correct responses the unannounced change in solution was made. Criterion for postshift solution was also 15 consecutively correct responses.

In order to match the conditions of other experiments which have reported R-IRD shift differences, a card sorting task and relatively brief instruction was used. In both respects, the procedure differs from Johnson's study in which Ss were given detailed instructions on how to solve the problem and were required to respond to slides projected on a translucent screen by pressing one or two buttons.

\section{Results and Discussion}

Since there was no difference between analyses of variance on errors and trials to criterion, only error data are presented. Ananlysis of preshift performance showed Dimensions, $F=13.32$, $\mathrm{df}=1 / 96$, and Problem, $F=18.97$, df $=1 / 96, p<.01$, to be the only significant sources of variance. The increase in task difficulty as the number of irrelevant dimensions increased from 2 to 4 has been found in several previous experiments (Bourne \& Haygood, 1959; 1960). The Problem effect reflects the greater difficulty of sorting on the basis of size rather than color for the particular stimulus deck used in this experiment.

Figure 1 presents mean errors to criterion in the postshift phase for the $R$ and IRD shifts with cue cards Present and Absent and with 2 and 4 irrelevant

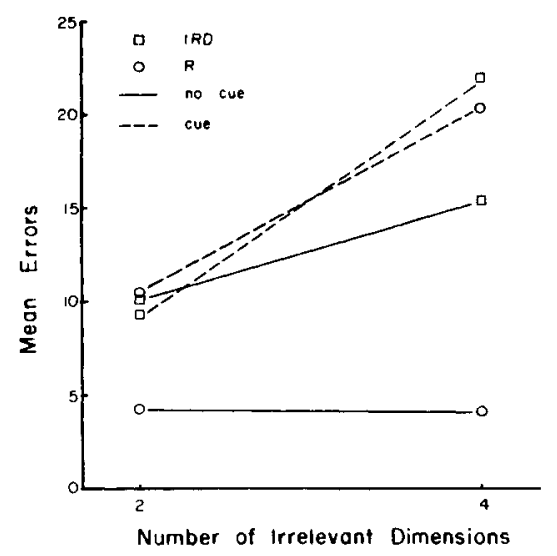

Fig. 1. Mean errors to Criterion on post shift phase for $R$ and IRD shifts with and without cue card over 2 and 4 irrelevant dimensions. 
dimensions. An analysis of variance indicated no difference between Problems or among Methods of Presentation. The effect of number of irrelevant Dimensions, however, was highly reliable, $\mathbf{F}=\mathbf{1 1 . 5 0}$, $\mathrm{df}=1 / 96, p<.01$. This effect held for both shift types when cue cards were present, but only for an IRD shift when cards were absent. As a result the overall Shift effect was small, $F=3.81$, $d f=1 / 96, p<.10$, while Cue Card condition, $\mathrm{F}=10.34, \mathrm{df}=1 / 96, \mathrm{p}<.01$, and the interaction of Cue Card and Shift, $F=4.11$, df $=1 / 96, p<.05$ were statistically significant. Since Ss were required to sort against the cue card in $\mathrm{R}$ shift problems an adverse effect was expected. That a similar effect was obtained for the IRD shift with 4 irrelevant dimensions was not anticipated in view of the fact that cue card(s) were consistent with post shift solution.

The primary aim of this experiment was to determine whether either of two procedural variables-availability of cue cards and method of problem presentationcontributed to the failure to find a difference between $R$ and IRD shifts in Johnson's (1965) experiment. The results show a significant $R$-IRD difference, $F=15.93$, $d f=1 / 50, p<.01$, for all three methods of presentation only when no card was present. But cue cards were not available to Ss in Johnson's experiment. Therefore, while it is interesting and important to note that the availability of cue cards may reduce or eliminate differences in shift difficulty, other factors must have contributed to the atypical results in Johnson's study. Amount of preshift training, instructions, and task format are left as possible variables to be investigated.

\section{References}

Bourne, L. E., Jr., \& Haygood, R. C. The role of stimulus redundancy in concept identification. J. exp. Psychol.. 1959, 58, 232-238

Bourne, L. E., Jr., \& Haygood, R. C. Effects of intermittent reinforcement of an irrelavant dimension and task complexity upon concept identification. J. exp. Psychol, 1960, 60, 371-375.

Johnson, P. J. Some factors affecting solution of transfer in concept identification problem. Ph.D. Thesis, University of Colorado, 1965

Kendler, H. H., \& Kendler, T. S. Vertical and horizontal processes in problem solving. Psychol. Rev., 1962, 69, 1-16.

\section{Note}

1. This research was undertaken in the Behavior Research Laboratory, Institute of Behavioral Science, University of Colorado and is Publication 61 of the Institute. The work was supported by Grant MH 08315 from the National Institute of Mental Health, U. S. Public Health Service and by Grant GB 3404 from the National Science Foundation. 